\title{
Pobreza e câncer do colo do útero: estudo sobre as condições de vida de mulheres com câncer do colo do útero avançado em tratamento no Hospital do Câncer II - Instituto Nacional do Câncer - Rio de Janeiro
}

Poverty and cervix cancer: A study about life conditions of women with advanced cervix cancer on treatment at the Hospital do Câncer II - Brazilian National Cancer Institute - Rio de Janeiro

Autora: Celia da Silva Ulysses de Carvalho Orientadora: Dra. Ana Maria de Vasconcelos

\section{Resumo}

Este estudo objetiva analisar as condições de vida de mulheres com câncer do colo do útero avançado em tratamento no Hospital do Câncer II do Instituto N acional de Câncer - Rio de Janeiro. Estas mulheres pertencem aos segmentos menos favorecidos da classe trabalhadora, o que as coloca entre as mais vulneráveis aos riscos de desenvolvimento dessa neoplasia. 0 estudo teve um caráter exploratório, combinando uma análise qualitativa e quantitativa, objetivando uma aproximação maior à realidade de vida dessas mulheres. Foram realizadas 63 entrevistas com mulheres com câncer do colo do útero em estadiamento II, III e IV. Tomamos como referencial teóricometodológico a abordagem teórico-crítica, compreendendo o câncer do colo do útero no contexto sócio-histórico como uma expressão da questão social e seu reflexo no cotidiano das classes subalternas. 0 estudo evidenciou que o cotidiano dessas mulheres apresenta limites concretos que dificultam o controle de sua saúde e a prevenção de doenças, e neste caso, do câncer do colo do útero como rotina em suas vidas. Limites que são potencializados pelas deficiências dos serviços de saúde e pela forma como estão estruturados na atenção à saúde da mulher. 0 estudo aponta questões relevantes que permitem compreender o diagnóstico tardio da doença. Q uestões que devem ser consideradas no desenvolvimento das ações de prevenção e controle do câncer do colo do útero, tais como: a precariedade das condições de vida dessas mulheres; sua cultura acumulada sobre saúde; o conhecimento limitado sobre prevenção de doenças e, especificamente, do câncer do colo do útero; a estrutura dos serviços de saúde e a qualidade da atenção profissional destinada às mulheres. 